Théologiques

Théologiques

\title{
La résistance des rites traditionnels dans l'Afrique moderne
}

\section{Éric de Rosny}

Volume 4, numéro 1, mars 1996

Les Rites : céder en résistant

URI : https://id.erudit.org/iderudit/602432ar

DOI : https://doi.org/10.7202/602432ar

Aller au sommaire du numéro

\section{Éditeur(s)}

Faculté de théologie de l'Université de Montréal

\section{ISSN}

1188-7109 (imprimé)

1492-1413 (numérique)

Découvrir la revue

\section{Citer cet article}

de Rosny, É. (1996). La résistance des rites traditionnels dans l'Afrique moderne. Théologiques, 4(1), 57-73. https://doi.org/10.7202/602432ar

\section{Résumé de l'article}

Contrairement à certains pronostics, la modernité ne fait pas disparaître les rites traditionnels en Afrique. À partir du cas significatif d’un médecin camerounais qui se fait soigner " à l’indigène ", E. de Rosny réfléchit sur les causes de la vitalité de la vie rituelle dans les sociétés africaines aujourd'hui. Il montre qu'en deçà de raisons historiques, thérapeutiques et même culturelles, il convient de se placer au niveau anthropologique pour comprendre cette longévité. C'est dans sa conception de l'homme centrée sur le corps et la relation au groupe que l'Africain puise son besoin persistant d'une vie rituelle. Dans ce domaine, il peut donc mieux résister qu’un Occidental. 
Théologiques 4/1 (1996) 57-73.

\title{
La résistance des rites traditionnels dans l'Afrique moderne
}

\author{
Éric DE ROSNY \\ Faculté des sciences sociales \\ Université catholique d'Afrique Centrale \\ Yaoundé
}

\section{RÉSUMÉ}

Contrairement à certains pronostics, la modernité ne fait pas disparâtre les rites traditionnels en Afrique. À partir du cas significatif d'un médecin camerounais qui se fait soigner "à l'indigène ", $E$. de Rosny réfléchit sur les causes de la vitalité de la vie rituelle dans les sociétés africaines aujourd'hui. Il montre qu'en deçà de raisons historiques, thérapeutiques et même culturelles, il convient de se placer au niveau anthropologique pout comprendre cette longévité. C'est dans sa conception de l'homme centrée sur le corps et la relation au groupe que l'Africain puise son besoin persistant d'une vie rituelle. Dans ce domaine, il peut donc mieux résister qu'un Occidental.

À la différence de l'Occident, l'Afrique - entendons celle qui commence au sud du Sahara, loin de la blanche mer Méditerranée - ne trouve pas de peine à définir ce qu'est pour elle la modernité. Celle-ci coincide avec l'irruption de l'Occident dans son histoire. Alors que la modernité en Europe et en Amérique du Nord est née comme un enfant après une longue gestation, en gardant les traits de ses parents naturels, la modernité en Afrique fait figure de bâtard, même si elle est un objet de fierté. Elle a été comme imposée à ce continent de l'extérieur et non sans douleur, dans une histoire relativement récente coüncidant avec le début de la colonisation, à partir du milieu du XIX ${ }^{e}$ siècle. Aussi est-il naturel en Afrique d'opposer la modernité à la tradition, cette dernière désignant le mode de vie social antérieur à la venue des colonisateurs. 
Pratique et fondée sur l'histoire, cette distinction trop radicale ne rend compte que superficiellement de la réalité. L'objet de cet article est de montrer, par le biais significatif de l'étude du rite, avec un exemple à l'appui, que la tradition perdure dans la modernité au point de la marquer de son sceau, tout autant qu'elle est elle-même transformée par cette dernière. Cette union, illégitime à son origine, fait que des rites, même les plus anciens - ou justement parce que ce sont les plus anciens - gardent une fonction utile dans la vie moderne au point d'en faire partie. Quelle est la raison de cette alliance non pas contre nature mais contre l'histoire?

\section{Visages de la modernité en Afrique}

\section{La ville}

Le trait le plus saillant du visage de l'Afrique moderne est "la ville». Non pas la ville moyenne qui tarde à se développer - phénomène bien connu des sociologues - mais la métropole. Avant la colonisation, en effet, il n'y avait pas à proprement parler d'importante concentration urbaine dans l'Afrique au sud du Sahara, sinon les villes construites par un Islam venu du nord, Ibadan, au Nigéria, que l'on cite comme une exception, et l'une ou l'autre agglomération dans le sud-est du continent. Elles ont été peuplées par des migrants. L'afflux des jeunes paysans vers la ville, croyant se libérer ainsi du carcan des coutumes villageoises, c'est-àdire de la tradition, se poursuit à l'heure actuelle, malgré l'absurdité économique que cette vague représente. En trente ans, le pourcentage des citadins est passé dans ces pays de $17 \%$ à $50 \%$ de la population globale, compte tenu de variations plus ou moins prononcées selon les régions.

À Douala, grand port du Cameroun en Afrique Centrale, d'où je tire l'exemple que je vais développer, la population a septuplé en trente ans, passant de 200000 habitants en 1957, quand j'y suis arrivé, à 1500000 aujourd'hui, selon les sondages les plus crédibles ${ }^{1}$. Certes, la croissance

1 Le Cameroun, colonie allemande à la fin du XIX $\mathrm{X}^{\mathrm{e}}$ siècle, passe sous mandats français et britannique en 1919 et devient indépendant en 1960. Situé entre le Nigéria, en commençant par l'Est, le Tchad, la Centre Afrique, le Gabon, le Congo et la Guinée équatoriale, il est bordé, pour boucler le tour, par l'Océan Atlantique au Sud-Est. Il a une superficie semblable à celle de la France et possède onze millions d'habitants. Deux grandes villes se disputent la suprématie: Yaoundé, la capitale administrative et Douala, la capitale économique qui est en même temps le port principal. La population est à majorité chrétienne (catholiques et protestants presque à égalité) dans les deux 
du trafic du port, passant de un million de tonnes en 1960 à sept millions en 1995 peut expliquer en partie l'attirance de la ville pour une même proportion de jeunes paysans séduits par l'espoir d'un salaire à la fin du mois. Ce salaire, même hypothétique, les fascine comme un symbole de modernité et de liberté et les attire vers la ville. Mais la principale raison est qu'ils ne veulent plus rester d'éternels mineurs au village, où la hiérarchie des âges et des générations reste, en se durcissant, la règle sacrée de la distribution du pouvoir et de l'argent. Pourtant leur manière de se comporter en ville rappelle leurs attaches paysannes: le mépris des horaires, le goût des fêtes saisonnières, la nostalgie de l'ambiance du village, le sens des rites sacrés. La tradition les habite encore!

L'État

Le second trait du visage de la modernité, second dans l'ordre de la visibilité mais non pas en importance, est la "notion d'État». Je dis bien «notion», tant est encore grande la distance entre elle et la réalité. Ces pays, indépendants pour la plupart depuis les années 60 , ont reçu des frontières toutes faites, un modèle de fonctionnement d'État tout trouvé, sans avoir connu la lente gestation des nations européennes qui les ont colonisés. Derrière une façade d'État, les rouages de la vie sociale et politique demeurent claniques le plus souvent, c'est-à-dire inspirés encore par des règles de relations familiales, dont la tradition reste souterrainement l'inspiratrice.

Cela s'est vérifié à Douala lors d'une grève générale de la population, appelée "opération ville morte", qui a duré cinq mois, entre mai et octobre 1991, pour protester contre le refus du gouvernement d'instaurer un large débat national de type démocratique. On aurait pu s'attendre à ce que ce mouvement, supporté par la majorité de la population, serve de ressort à l'avènement d'un État conforme à une volonté nationale. Il n'en a rien été. L'« opération ville morte " s'est éteinte d'elle-même, faute d'une véritable dynamique. L'analyse des événements a fait apparaîrre, comme raison profonde de l'échec, la prédominance du caractère décidément "familial " de la société, malgré les bouleversements provoqués par la vie urbaine. Il est bien connu qu'une société de type familial, si étendue qu'elle soit, au point d'être appelée une "ethnie", ne peut pas être le support d'une action révolutionnaire déterminante.

villes et tout le Sud. Au Nord se trouve une minorité de musulmans. Les « religions traditionnelles" sont surtout vivantes dans les montagnes de l'Ouest. 


\section{La Religion}

Si paradoxal que cela puisse paraittre en comparaison avec la situation en Occident, le troisième trait de la modernité se trouve être "la religion ". À vrai dire, le mot désigne l'Islam et le Christianisme et non pas ce qu'il est aujourd'hui coutume d'appeler "la religion traditionnelle". C'est que le terme même de religion ne semble pas avoir d'équivalent dans les langues africaines, à moins qu'on ne l'ait forgé après coup. Rien d'étonnant à cela. Le concept de "religion" suppose en effet l'existence d'autres secteurs de la vie personnelle et sociale qui n'en dépendent pas, autrement pourquoi employer un terme propre? Alors que la vie tout entière était - et le reste encore concrètement, comme nous le verrons — imprégnée de « religion".

Ainsi ai-je mis du temps à comprendre la raison de la présence du mot «ebassi ", donné pour "religion", dans le vocabulaire de la langue douala que j'ai apprise. Selon moi, il n'avait pas de raison d'y figurer. Engagé d'abord sur de fausses pistes, j'ai fini par découvrir au cours de mes recherches que le mot signifiait en réalité «mouchoir». Dans le dictionnaire douala-français, établi par le Révérend Pasteur P. Helmlinger (Éditions Klincksieck, Paris 1972), le premier sens donné au mot est bien "religion" mais le second "mouchoir". L'explication est amusante. C'est que le premier missionnaire au Cameroun, le Pasteur Alfred Saker, en 1845, exigeait, conformément à la consigne de l'apôtre Paul, que les femmes aient la tête couverte à l'église. Faute de chapeau, les premières catéchumènes posaient sur leur tête un mouchoir en guise de fichu ou de foulard! Les observateurs doualas du phénomène, non encore chrétiens à cette époque, désignaient ainsi, ironiquement sans doure, la nouvelle religion.

Bien perspicace celui qui saura démêler à la vue du comportement journalier des citadins ce qu'ils doivent à la modernité et ce qu'ils retiennent de la tradition, ce qui vient de la ville et ce qu'ils ont apporté avec eux du village, la part des religions nouvelles et celle des croyances ancestrales. Cette impression d'amalgame, l'observateur la ressent particulièrement à la vue de la vie rituelle: à Douala, grand-messes de rite latin démesurément prolongées; fêtes musulmanes célébrées avec faste; pullulement de groupes de prière, reconnus par les Églises ou considérés par elles comme sectaires; parades officielles lors des événements nationaux, redonnées indéfiniment à la télévision; quotidienne retransmission des visites faites au Président de la République par des grands de ce monde; les liturgies des matches de football... et, se maintenant toujours, malgré la concurrence, les célébrations rituelles héritées du passé. Tout se passe 
comme si la ville, l'État et la religion, expressions de la vie moderne, avaient comme exacerbé et non fait s'estomper le besoin inné d'une vie rituelle.

\section{Le docteur Manga}

Le cas que j'ai choisi de présenter en guise d'exemple est celui d'un homme que l'on peut qualifier de "moderne" selon les critères que je viens de proposer. Originaire de la ville de Douala, il y a suivi jusqu'au baccalauréat le parcours de l'enseignement secondaire. Parti en France pour des études supérieures, il est revenu au pays en qualité de médecin, profession qu'il exerce toujours à Douala. Au cours de l'opération "ville morte " en 1991, il s'engagea dans l'opposition politique et occupe mainrenant un poste de responsabilité dans un parti. La religion qu'il pratique sans ostentation mais avec régularité est de confession protestante. Pourtant il se soumettra à des rites hérités de la coutume de sa famille avec une ferveur dont j'ai été le témoin. Je proposerai une explication pour cette cohabitation en un même homme de la tradition et de la modernité.

\section{Les visiteurs}

Manga vient me rendre visite, un après-midi, accompagné d'un ami commun, au Centre de Rencontre que les Jésuites tiennent dans la banlieue de Douala. Il attend son tour dans une salle attenante au petit bureau où je reçois. En effet, depuis le début de ce que l'on appelle ici « la crise économique", bien des personnes ou des familles désemparées cherchent désespérément un guide, un devin, un "père spirituel ", en un mot un «médiateur» qui puisse leur indiquer la voie à suivre pour retrouver une santé et un bien-être mis à mal, selon eux, par une situation sociale bloquée. Connaissant mon itinéraire à la fois de religieux et de chercheur, qui combine à leurs yeux le pouvoir et le savoir, ils s'adressent à moi nombreux, après avoir frappé, je le sais, à d'autres portes, en justifiant ainsi leur démarche : «Nous venons vous demander une aide plutôt que d'aller chez un charlatan!" Le terme ambigu de "charlatan " pouvant désigner aussi bien l'authentique guérisseur traditionnel, appelé "nganga», que l'exploiteur de la crédulité populaire qui hante aujourd'hui les rues et les quartiers. L'émission de Radio-Douala que j'assure tous les vendredis soirs pour répondre aux lettres des angoissés du monde moderne, m'amène de nombreux visiteurs et visiteuses, seuls ou en famille. 


\section{L'angoisse}

Manga entre dans mon bureau précédé de cet ami commun que je connais depuis longtemps pour l'avoir tenu autrefois comme élève. Celui$\mathrm{ci}$, je l'appellerai Epessé. Ils sont de la même génération, frisent aujourd'hui la quarantaine et militent dans le même parti politique. Même corpulence, même taille moyenne. Tout les rapproche, y compris la peur que connaît Manga, non pas par un effet de contagion mais à cause de la solidarité communicante qui prévaut entre amis. Manga a été contacté un soir par un inconnu chargé d'une mission à son endroit, lui a-t-il semblé, et d'un message dont il n'a pas su démêler le sens. Il est resté sur le quivive durant l'entretien qui fut court, mais fut pris de tremblements, comme jamais il n'en avait éprouvés, une fois l'homme parti. Une bouffée de chaleur lui est montée des pieds jusqu'à la tête et a provoqué, m'explique-t-il, comme un dédoublement de sa personne. Depuis ce moment fatal, «il ne se sent plus en lui", "il n'est plus dans son corps", un sentiment qui le terrorise. Et, comme par voie de conséquence, il ne dort plus. Cet incident tombe d'autant plus mal que Manga doit se rendre bientôt dans la capitale du pays, Yaoundé, pour une importante réunion politique de son parti. Il se demande si ses ennemis ne veulent pas ainsi l'empêcher d'y participer. Epessé lui a conseillé de me consulter et a tenu à l'accompagner.

Après avoir écouté son récit, je lui dis simplement : "Vous êtes victime d'une agression! "Un psychiatre aurait-il donné raison aussi vite à l'interprétation que lui offrait le patient? J'en doute. Les symptômes de type paranoïaque l'invitaient plutôt à explorer la vie antérieure de Manga avant de se prononcer. Mais j'ai réagi comme je l'ai vu faire si souvent par les nganga, ces héritiers de la médecine traditionnelle sur la côte du Cameroun, qui, au dire des psychiatres eux-mêmes, soignent mieux qu'eux les brins de folie. J'ai choisi de parler et d'agir, comme je le fais d'habitude, en me glissant à l'intérieur du modèle de représentation que la victime se figure de son trouble, où le mal est né, s'est développé et, dans le meilleur des cas, pourra se résoudre. Manifestement le modèle auquel se référait Manga était celui de sa tradition qui m'est devenu familier et non pas celui de l'hôpital ou de son Église. À sa demande d'un conseil sur la marche à suivre pour se protéger et se libérer, je lui propose donc le rite traditionnel de l'Essa.

\section{Vers la tradition}

L'Essa a l'avantage d'être une cérémonie admise par tous. Elle s'apparente au célèbre palabre africain au cours duquel chacun proclame son 
innocence et sa volonté de venir en aide à la personne inquiète, créant autour d'elle une aura de sécurité et de solidarité. Ce sont des assises familiales, en quelque sorte, dont j'ai souvent remarqué l'efficacité thérapeutique. Le rite jouit d'un grand prestige dans les milieux coutumiers pour lesquels il reste un ressort majeur. Les Églises n'ont rien à y redire car il ne comporte pas, du moins explicitement, de références religieuses, ni non plus l'Administration ou l'État. Des trois éléments principaux de la modernité, c'est encore la ville, avec son bouleversement des relations sociales, qui rend difficile la tenue de l'Essa. En effet, la validité de la cérémonie dépend de la présence de notables de la famille chargés de la présider. Or, le pouvoir de ces chefs locaux a singulièrement perdu de sa force avec l'avènement de la hiérarchie moderne de l'Administration. Quand je rappelle à mes deux visiteurs cette exigence, ils sont embarrassés : dans la famille de Manga on ne peut plus trouver un représentant de la génération précédente qui fasse autorité! Je leur conseille alors de faire appel à une instance traditionnelle de substitution et de s'adresser à un nganga. J'en ai un à leur proposer que je connais depuis quinze ans: Bernard Nkongo.

\section{Un rite traditionnel en ville}

La description qui suit ne peut être ni détaillée ni complète. Elle est faite ici pour servir notre propos, à savoir l'étude de la persistance d'activités rituelles traditionnelles dans un contexte moderne. Aussi n'en livreraije que les principales étapes et, autant qu'elle m'est connue, leur signification.

\section{La préparation}

Il s'agit pour le nganga Nkongo de délimiter un espace où il sera le maître - sous le contrôle de ses ancêtres - le temps d'une nuit de traitement, et ceci en plein quartier populaire de Douala. Cette superposition d'un espace sacré à un espace profane a quelque chose d'insolite et d'étrange quand on voit passer à proximité des cyclistes et des promeneurs apparemment indifférents, qui vaquent à leurs occupations de citadins. Dans un environnement villageois, où se pratique encore ce rite, l'impression est au contraire celle de la coüncidence ou, du moins, de l'harmonie des espaces symboliques et réels. Pour tracer le contour de l'espace, Nkongo pose sur le sol, autour de la couretre qui sépare sa maison de la rue, une barrière de tiges mises bout à bout, appelées "ekoka bedimo " (canne des Morts). Puis, il projette dans tous les azimuts de la surface ainsi délimitée, d'impressionnantes bouffées de feu purificatrices et défensives, comme un halo rassurant de lumière dont il est le centre. 
Manga, Epessé, deux hommes âgés et moi-même sommes assis sur des petits bancs contre le mur de la maison, à l'intérieur de l'espace réservé. Il fait déjà nuit.

Nkongo a assisté pendant dix ans un célèbre nganga aujourd'hui décédé que j'ai vu exercer dans ce même endroit, quand les hautes herbes le recouvraient encore. Il est intéressant d'observer l'ancien apprenti reproduire à l'identique les mêmes gestes que son maître mais dans un cadre désormais urbain. Nkongo possède un atout, qu'il a mis longtemps à obtenir, pour avoir le droit d'exercer comme nganga: la double vue. Avec elle, il donne une grande importance à ses rêves dont le sens détermine son action: "J'ai vu Manga, m'a-t-il confié avant le traitement. Il était accompagné d'un homme suspect. Nous étions debout au-dessus d'un ravin. S'il fait un faux pas, il va tomber! Je lui ai donc dit que son affaire était grave! " Ces «quatre yeux " en activité de jour comme de nuit, et l'expérience acquise font de lui, à cinquante ans, le maître incontesté de la «médecine traditionnelle» dans son quartier. Il a fait trois années d'études de comptabilité, exercé de petits métiers comme celui de receveur dans une compagnie de transport urbain et il est devenu le secrétaire de la cellule locale d'un parti politique. Ajoutons à cette fiche d'identité qu'il est père de sept enfants tous nés à Douala, et l'on comprendra que Nkongo est un citadin à part entière, non pas un villageois égaré en ville.

\section{L'Essa}

Le docteur Manga est maintenant assis sur un tabouret, ne portant rien, hormis un petit caleçon, seul au centre de la courette devant une bassine pleine d'eau. C'est que le rite de l'Essa réserve une grande place aux ablutions. On dira même que le rite consiste essentiellement à « se laver ». Se laver non pas de ses propres souillures mais de celles que l'entourage, familial ou professionnel, a pu accumuler sur soi. Rares sont les allusions à la responsabilité personnelle de la victime. La distance d'avec la conception chrétienne de la culpabilité apparaît déjà ici. Mais avant de passer au bain d'eau, Manga sera déjà "lavé " par les paroles des assistants qui donneront sens au rite.

Ainsi, Nkongo invite chacune des personnes présentes à s'exprimer. À Epessé de commencer, bientôt suivi par les deux vieillards, garants de la tradition. Tout en articulant les mots sacrés, il postillonne sur le visage de Manga les fragments d'une graine, le «bongolo », qu'il a du mâchonner au préalable. Pas de parole sans son support tangible : "Que tout le mal qui t'entoure parte au loin! Quel que soit celui qui te barre le chemin, de jour comme de nuit, qu'il s'éloigne donc pendant que je suis en train de te 
laver! Je demande que tout ce qui te concerne soit désormais en ordre!" Discours prononcé, bien évidemment, en langue douala. Quand mon tour arrive, je me récuse. À Nkongo, à qui je me devais plus tard de justifier mon attirude, $j$ 'expliquerai que je ne connaissais pas assez Manga pour m'engager ainsi, sans pour autant m'opposer à un rite que je lui avais conseillé. Sans doute, un certain instinct chrétien m'avait aussi retenu. Le tour des intervenants terminé, Manga est invité à s'asperger d'eau, ce qu'il fait avec conscience et énergie.

\section{Le retour à l'ordre}

La cérémonie de l'Essa proprement dite est terminée. Mais, animée par un nganga, elle ne peut qu'être prolongée à sa manière. Guérir une personne, c'est la situer à nouveau à sa place dans l'ordre cosmique et humain dont elle a été écartée par les maléfices d'autrui au risque de voir la mort. Son angoisse n'en est-elle pas le signe? Pour ce faire, Nkongo va d'abord réintégrer son patient dans l'ordre de la nature par la grâce des rites. Dans la bassine pleine de cet élément vital qu'est l'eau, marinent neuf herbes médicinales et neuf écorces majeures de la tradition. Neuf est le chiffre de la complétude dans l'univers culturel bantou. Je les ai identifiées quand Nkongo faisait sa préparation. Depuis plusieurs années nous travaillons tous les deux avec une équipe pour établir un bilan de la pharmacopée en langue douala et avons réussi à consigner pas moins de quatre cent cinq plantes différentes à usage médicinal. La présence de cette végétation baignant dans l'eau du bain a donc, en l'occurrence, une efficacité symbolique, à condition de donner à cette expression toute sa portée thérapeutique.

Place maintenant au règne animal. Nkongo s'empare d'un plantureux coq roux et le frotte avec application sur le corps de la victime, en fredonnant des mélodies incantatoires tirées du répertoire des nganga. Au village ou "en brousse ", il aurait chanté à pleine voix, mais le caractère intime de la cérémonie eu égard à la personnalité du patient connu dans le quartier, exigeait une certaine discrétion. "Toi le coq, ordonne Nkongo, veuille éloigner de Manga ses ennemis, qu'ils soient hommes ou femmes, enfants ou vieillards, Blancs ou Noirs! Éloigne-les à partir de l'instant où je te frotte sur lui! » J'avais demandé que la cérémonie ne donne pas lieu à l'immolation d'un animal. Comme j'en étais l'initiateur, je tenais à éviter des actes rituels en flagrante contradiction avec la logique chrétienne. Manga et Nkongo n'étaient-ils pas protestants, Epessé catholique et moi prêtre de surcroît? Et je n'avais pas de raison de suspecter leur appartenance à une Église chrétienne. Jésus-Christ n'avait-il pas mis fin par son "sacrifice volontaire " à l'antique série des immolations 
d'animaux? Le coq ne fut donc pas saigné. Mais je ne suis pas sûr que la coutume d'immoler un animal n'ait pas été respectée quand Nkongo cassa un œuf de poule sur le front de la victime! «Un œuf n'est-il pas (en puissance) mille poulets, m'avait dit une fois un nganga?»

Maintenant, Nkongo extrait neuf pièces de monnaie qu'il avait posées préalablement au fond de la bassine et les frotte sur le front de Manga : réconciliation de celui-ci avec l'univers moderne de l'argent! Avec de petits objers que je cesse d'énumérer ici car on en connaît maintenant la signification globale, il continue jusque tard dans la nuit à replacer son patient dans son univers habituel et normal qu'il a miniaturisé par ces rites. Une fois cet équilibre retrouvé, il peut s'attaquer à la cause de l'angoisse qui ne doit pas être recherchée ailleurs que dans son entourage immédiat, à savoir sa famille. J'avais pensé spontanément que Nkongo allait porter l'accusation sur les milieux politiques où s'activait dangereusement son client. C'était oublier un adage de la coutume: "Ton sorcier fait partie de ton corps! "Il faut entendre: "Celui qui te fait du mal est quelqu'un des tiens ». Aucun homme politique ne peut donc être atteint sans la complicité d'un membre de sa famille. C'est la famille qui se rappelle au bon souvenir et à l'attention des hommes d'État! Nkongo va signifier cela à sa manière. Il dépose le coq sur le crâne de Manga, de telle sorte que la bête s'y cramponne un temps, puis il l'en déloge par une bouffée de feu. Le coq s'enfuit vers la gauche, loin de la tête du patient: un signe, nous dira-t-il ensuite, que le coupable se trouve dans la lignée de la famille maternelle. À la différence des charlatans de ville, enclins à porter des accusations explicites à tort et à travers, Nkongo n'en dira pas plus, quoique sa double vue lui permettrait d'être plus précis. Il se contente ainsi d'orienter l'agressivité de la victime sans déclencher la zizanie familiale.

Très discrète aura été durant la cérémonie la référence aux "puissances invisibles", aux " esprits ", faisant éminemment partie de la cosmogonie traditionnelle. Les mots en langue ne manquent pourtant pas, précis et variés, et les premiers missionnaires n'ont pas eu de peine à puiser parmi eux pour traduire le nom du Dieu unique de la Bible. Ma présence, mais surtout l'appartenance au christianisme de tous les participants ont fait que le panthéon traditionnel n'a pas été publiquement évoqué. Sauf les ancêtres, puissances rendues profanes aujourd'hui, si l'on peut dire, et Dieu, invoqué selon le vocable adopté par les missionnaires, à savoir "Loba". Plusieurs fois Nkongo les a nommés en levant son visage vers le ciel obscur et nuageux qui surplombe la ville. Un compromis religieux qui donnait une plate-forme commune au culte traditionnel 
et au christianisme, sans que personne ne souhaite en éclaircir les composantes.

\section{Le verdict}

Peu avant l'aube, nous nous retrouvons tous dans le salon de la maison pour un repas que Manga a payé avec largesse, que l'épouse de Nkongo nous a préparé et que je suis invité à bénir. Il est sans valeur " rituelle " cette fois, suite à mon intervention, puisqu'il n'est composé ni d'un poulet ni d'une chèvre, que l'on aurait sacrifiés pendant la cérémonie, mais il a une saveur et une signification humaines manifestement appréciée de tous. Une fois restauré, Nkongo se lève pour affirmer avec autorité que le traitement s'est bien passé et que Manga peut se rendre sans crainte à Yaoundé le lendemain à l'occasion de l'importante réunion politique à laquelle il est invité, décision qui était l'enjeu de la cérémonie de l'Essa. Cependant, ajoute-t-il, Manga devra rester à l'écart de la salle de conférence sans y entrer. Qu'il se trouve un porte-parole qui puisse défendre en son nom ses intérêts politiques! Le verdict de Nkongo n'est pas extravagant car cette forme de stratégie est habituelle dans les milieux politiques. Et nous nous séparons au petit jour. Manga, rasséréné, autant que je puisse m'en rendre compte, suivra à la lettre les instructions.

\section{Le discernement}

Pourquoi n'ai-je pas adressé Manga à un psychiatre, peut-on se demander? À vrai dire cette spécialité médicale est faiblement représentée à Douala : un seul psychiatre débordé pour un million et demi d'habitants, dont on peut aisément deviner que les conditions de vie agressent et ébranlent la santé mentale! Sur les rues principales, et surtout dans les carrefours, il n'est pas rare de côtoyer des fous et de folles, hirsutes et nus, trouvant au milieu d'une foule bonne enfant un climat chaleureux qui trompe leur isolement. Mais Manga connaît comme un chacun l'existence du psychiatre et ne s'est justement pas adressé à lui. C'est qu'une forte bouffée d'angoisse, un psychiatre le reconnaîtra, peut entraîner un phénomène de "régression mentale » tel que le sujet éprouve le besoin incoercible de retourner, pour ainsi dire, dans le sein maternel de sa culture et sous ses formes d'expressions rituelles les plus anciennes. $\grave{A}$ ce niveau, le sauveur sera le nganga qui participe de la même culture, tandis que le psychiatre, même s'il est africain, comme celui de Douala, se trouvera plutôt démuni car il doit son savoir à une autre culture.

Pourquoi n'ai-je pas donné droit, peut-on encore se demander, au caractère chrétien de Manga et ne l'ai-je pas adressé, par exemple, à l'un 
des nombreux groupes de prière du Renouveau charismatique qui se réunissent le soir dans les églises de la ville, catholiques et protestants confondus? Si la prière n'est pas une médecine, comme je le répète chaque jour à mes visiteurs, n'a-t-elle pas cependant un «effet " salutaire sur la santé? Ou pourquoi ne lui ai-je pas imposé moi-même les mains audessus de la tête, comme il m'arrive de le faire de plus en plus souvent, depuis que j'ai découvert l'impressionnant effet du geste liturgique sur la personne et ses accompagnateurs? Mais j'ai eu l'intuition que l'homme venait chercher chez moi un encouragement ou même une forme d'autorisation avant de se tourner vers sa tradition, lui dont certains éléments de culture étaient désormais chrétiens et "modernes». Français et prêtre, je représentais sans doute pour lui comme une garantie de fidélité à d'autres aspects de sa personnalité.

Avec quelle intention l'ai-je fait? Dans le seul but, je puis répondre, de l'aider dans l'immédiat à vaincte son angoisse. Mais je reste convaincu que la cure du désenvouteur ne peut pas être un modèle thérapeutique d'avenir à proposer à une société moderne, même si elle lui est encore et pour longtemps nécessaire. Autre est la prise en charge d'une personne en détresse, autre est l'objectif général à poursuivre. Ma réserve au sujet des sacrifices et ma discrétion au cours du traitement n'étaient-ils pas ma façon de le signifier à Manga? A-t-il perçu le message? Sans doute pas sur le moment, et je me promets de le lui faire entendre au cours d'un autre entretien, une fois qu'il sera sorti de son état de marasme, lorsqu'il éprouvera le besoin à nouveau de "revenir en arrière " et de prendre en ma compagnie un certain recul critique sur ces événements. $\AA$ chaque nuit suffit sa peine!

\section{Le soubassement anthropologique des rites traditionnels}

J'ai apporté au débat sur l'alliance des rites et de la modernité le cas de Manga parce qu'il m'est apparu, pour l'avoir vu se répéter avec de multiples variantes, comme significatif d'une société. " $\AA$ bien des égards, écrit Le Guérinel, les structures mentales évoluent plus lentement que les structures sociales. Le sujet se trouve ainsi affronté à la tâche d'organiser une nouvelle mise en scène du désir, alors que, paradoxalement, les symptômes continuent à garder la forme des anciens modèles culturels " (Le Guérinel 1987). Cet écart certain entre les «structures sociales" et les "structures mentales" apparaît pourtant, dans le cas des sociétés africaines, moins "paradoxal " qu'ailleurs et plus facilement explicable. Les structures d'un État moderne, on le sait, reposent à l'origine sur une philosophie grecque qui a fait la fortune de l'Occident et s'est imposée à l'Afrique, tandis que les structures mentales restent basées sur une vision 
de l'homme issue de la sagesse bantoue. C'est à ce niveau philosophique, me semble-t-il, plus éclairant que celui de la sociologie ou de l'histoire, que nous devons décidément nous situer si nous voulons non seulement évaluer la largeur du décalage existant entre les structures sociales et les mentalités, comme le suggère Le Guérinel, mais aussi comprendre les raisons de la capacité de résistance des rites traditionnels dans une société moderne.

\section{La notion de corps}

La présence dans les langues africaines de termes qui correspondent littéralement ou approximativement à l'âme et au corps de la philosophie grecque, qui a marqué la théologie chrétienne, ne doit pas tromper : ici, c'est le corps qui englobe l'âme, pour ainsi dire, et non l'âme qui " informe » le corps. Cette différence d'optique peut s'observer déjà dans la manière de traiter les enfants, qui est si révélatrice de la conception que se fait de l'homme une société :

Tous les auteurs décrivent le remarquable accord somatique entre la mère et l'enfant au moins jusqu'au sevrage : corps à corps presque continuel que ce soit par le portage au dos ou par les manipulations corporelles. Mais aussi accord par la voie du sein qui est à la disposition entière du nourrisson. On a insisté sur la sécurité de base que procure cette attitude très gratifiante de la mère... Et ce type de contact qui se prolonge après le sevrage, se transformera progressivement en jeux de corps entre enfants. Aussi bien tout cela contribuera à donner à l'image du corps de l'Africain quelque chose de spécifique dont il ne se départira jamais, même dans les avatars que subira cette image dans les troubles psychosomatiques ultérieurs. (Lehman 1969)

Puis-je illustrer cette réflexion par une anecdote? 11 m'est arrivé une fois d'introduire dans une famille une assistante sociale française en visite à Douala. Un enfant en bas âge pleurait et sa mère n'avait de cesse que de le calmer en satisfaisant ses moindres désirs. "Laissez donc pleurer cet enfant le jour, conseilla-t-elle à la mère, forte de sa compétence, sinon il va crier toute la nuit! "Malgré mon ignorance en matière d'éducation d'enfants, j'ai cru bon de faire remarquer à l'assistante sociale qu'on ne pouvait pas changer une habitude - à savoir que le bébé doit être traité comme un perit prince jusqu'au sevrage - sans perturber la cohérence de son apprentissage et, à travers elle, une manière de vivre en société... 
Le corps représente la personne dans son entier. J'ai commencé à en prendre conscience le jour où un sage douala m'a donné le conseil suivant avant un voyage : "Prends bien soin de ton corps (nyolo)! ", plutôt qu'un "Fais attention de ne pas perdre ton âme $(e d i) !$ " Il voulait simplement me recommander de veiller à ma santé. C'est que la maladie figure non seulement la "dégradation du corps" (mbeu'a nyolo), ce qui ne nous surprend pas, mais la «division du corps » en une partie visible devenue comme une carcasse et une autre partie invisible où se réfugie l'énergie. La santé coïncidera avec la réunification des deux parties, de telle sorte que la personne ne fasse plus à nouveau qu'un seul corps. Ainsi cette plainte significative trouvée dans la lettre d'un enseignant, à laquelle je devais répondre sur les ondes de Radio-Douala: "Mon Père, je souffre beaucoup. Je ne suis plus qu'une carcasse sans énergie et sans tonus. Le plus gênant dans tout ça, c'est que je suis conscient et lucide "par ailleurs ». Je connais beaucoup de choses mais je n'agis pas... Il y a comme une force invisible qui me retient et qui me rend lymphatique et flottant dans ma carcasse. " À la différence de la conception grecque du composé humain, où l'âme est un principe "immatériel » et le corps "matériel ", la césure se fait ici au niveau de "l'invisibilité " et de la "visibilité ». La distinction n'est pas sans conséquence : la corporéité de l'homme ne sera jamais escamotée même si elle échappe en partie aux.yeux ordinaires.

\section{La notion de relation}

Mais un corps ne peut être perçu ni donc pensé sans sa relation avec les autres corps. La "relation interpersonnelle " que l'on pourrait appeler aussi bien la "relation intercorporelle " doit être considérée, si l'on veut comprendre le comportement des Africains, comme un élément constitutif de leur identité. L'individu, et davantage encore l'individualiste, est pris pour un personnage inquiétant dans la société traditionnelle parce qu'il se comporte comme s'il pouvait se passer du groupe pour exister et qu'il met en question son unité. Le terme le plus adéquat pour désigner en langue française ce que l'on entend ordinairement par "sorcier " ne serait-il pas celui d' 'individu ", c'est-à-dire l'homme ou la femme qui prétendrait profiter du groupe à son avantage personnel sans l'accord du clan? Aussi ne faut-il pas s'étonner que la "sorcellerie " prolifère en ville et qu'un sage m'ait déclaré : "Il n'y a jamais eu autant de sorcellerie qu'à Douala aujourd'hui!»

En effet, la société moderne mise sur la compétition, la concurrence, la promotion personnelle dès l'école, toutes valeurs ou considérées comme telles qui renversent l'idée que l'on se faisait de l'homme : un être éminemment social. Mais comme ces "valeurs" ou, si l'on préfère, ces res- 
sorts de la modernité échouent à faire vivre les gens heureux, par le fait d'institutions qui tardent à se mettre en place, la cohésion familiale peut garder sa force. Quand je suis arrivé à Douala en 1957, il était fréquent d'entendre dire que la "grande famille africaine " disparaîtrait bientôt au profit de la famille cellulaire, dont l'Occident offrait un modèle. Près de quarante ans plus tard, c'est le contraire qui s'offre à nos yeux : la grande famille continue de servir de référence, tandis que la vie du couple avec enfants manque de stabilité. Comme me le disait de façon abrupte un père de famille en instance de divorce: "On peut se séparer de son épouse, mais on ne divorcera jamais de sa famille! " Cette résistance de l'appartenance ethnique ne s'explique pas seulement par les déficiences de la société moderne dans sa phase actuelle, elle est soutenue par la conception que l'on se fait traditionnellement de l'homme comme «être-enrelation-corporelle $»$.

\section{La nécessité des rites}

Les brèves analyses précédentes permettent-elles de comprendre pourquoi les rites font partie intégrante de la vie des Africains et ne sont pas seulement une expression culturelle gratuite? Que l'on veuille bien me suivre. Si l'on se perçoit comme «corps" et que la « relation" aux autres corps est pour soi un élément vital, comment pourrait-on vivre sans rites? Un rite est un ensemble de gestes visibles par lesquels un groupe reste en communion ou retrouve son unité. Il y faut donc un support sensoriel et l'appartenance à une communauté, - et quoi de mieux que le corps, et quoi de plus fort que les liens familiaux? Étant donné la conception que les Africains se font du composé humain, comment pourraient-ils faire l'économie des rites dans leur existence? À contrario, le malaise rituel du monde occidental ne peut-il pas s'expliquer en son fond par la persistance d'un vieux contentieux d'avec le corps qu'il doit davantage à ses origines grecques qu'à l'héritage biblique, entraînant, par voie de conséquence, une montée de l'individualisme?

Pour reprendre l'expression de Lévy-Strauss qui s'est imposée en sciences humaines mais qui était depuis longtemps employée par les théologiens à l'attention des sacrements, les rites sont des "symboles efficaces" de la vie en communauté. Leur pouvoir ou leur portée correspond à leur capacité de mobiliser l'être même de la personne en signifiant «matériellement » l'appartenance de son corps - c'est-à-dire de sa personnalité - à une communauté hors de laquelle rôde la mort sociale. C'est pourquoi les rites ne sont pas de l'ordre de la gratuité mais de la nécessité. Rien de plus affligeant - et en même temps de significatif que de voir se dérouler les danses dites folkloriques les jours de grandes 
fêtes sur les places publiques des villes: ce sont des parodies de rites mais aussi les signes de leur résistance dans le contexte le plus étranger qui soit à la tradition.

Par contre les rites thérapeutiques des ngangas gardent, quand ils sont pratiqués par d'authentiques héritiers de la tradition, la capacité de mobiliser les énergies de leurs patients, comme ce fut le cas pour Manga. Le secret de leur efficacité est d'intervenir à un moment crucial. Quand les personnes sentent se dégrader à la fois leur corps visible (symptômes de la maladie) et leur réseau de relations (symptômes de l'isolement social), ils voient venir la mort. Ce sont les termes de Manga: "Je ne suis plus en moi, je ne me sens plus dans mon corps ". L'art du nganga est de reconstituer l'unité du corps et des relations sociales du plaignant pour lui redonner vie par l'effet salvateur du rite. Il fait usage de gestes et de paroles mais emploie aussi des plantes médicinales qui revigorent la "carcasse", une activité complémentaire que le cas de Manga ne m'a pas donné l'occasion de mettre en valeur. Olivier Marc a conclu par quelques mots bien choisis une présentation d'un traitement traditionnel que je venais de faire à Paris aux membres de l'Association jungienne de psychiatrie: "Alors que par la morale notre civilisation impose encore le refoulement de la vie pulsionnelle, l'Afrique ne cesse collectivement de tenter d'intégrer ces grandes forces qui nous font peur. C'est peut-être grâce à cela que les guérisseurs camerounais font preuve d'une étonnante connaissance du jeu des inter-relations sociales et familiales".

\section{Conclusion : les rites et l'Église}

Les voyageurs occidentaux de passage dans nos régions nous font part souvent de leur étonnement devant l'engouement des Africains pour la liturgie chrétienne: les églises sont pleines, tandis que celles de leur propre pays connaissent une singulière et préoccupante perte de pratique. L'incontestable ferveur liturgique des fidèles, leur aptitude à chanter au rythme de leur corps, leur prédilection pour les longues cérémonies peuvent faire rêver, elles ne doivent pourtant pas tromper : la pratique régulière n'est pas si élevée qu'elle le paraît.

Un exemple : à la demande de l'évêque de Douala, j'ai entrepris en 1991 une évaluation de la vie de l'Église. Il a fallu que le clergé se soumette à l'évidence des chiffres : pas plus de 15 à $20 \%$ de catholiques fréquentent nos églises le dimanche. Le pourcentage est probablement plus faible encore dans les lieux de culte protestants. À cela plusieurs explications peuvent être données : l'exiguïté et le trop petit nombre des édifices religieux qui obligent des fidèles à rester debout tout au long de l'office ou 
les décourage de s'y rendre; le rythme par trop régulier de l'obligation de la messe tous les dimanches, tandis que le tradition ne rassemblait la population qu'à certaines périodes de l'année; la lente augmentation du nombre des prêtres de paroisse détenteurs de l'autorité et du pouvoir d'initiative, moins nombreux qu'en Occident, quoiqu'on en dise, relativement au pourcentage des baptisés; et la concurrence croissante et significative des sectes et des Églises indépendantes, plus libres que les grandes Églises de laisser s'exprimer ou se défouler l'affectivité de leurs membres.

Mais la cause principale du défaut de pratique est à imputer, me semble-t-il, au facteur temps. L'enchantement visible des Africains pour la liturgie chrétienne qui frappe tant les observateurs, sans entraîner par là même une pratique régulière et massive viendrait surtout du caractère relativement récent de la première annonce de l'Évangile, tout juste un siècle dans la plupart des pays du sous-continent. À l'occasion de la célébration en 1991 du centième anniversaire de l'arrivée des premiers missionnaires au Cameroun, les évêques, fils du pays en grande majorité, n'ont pas manqué d'en appeler à une deuxième évangélisation pour une conversion en profondeur, en soulignant le caractère superficiel de la foi populaire.

Il n'en demeure pas moins que le goût et même le besoin incoercible de rites sacrés fournissent un terrain de choix pour la catéchèse. Cette prédisposition profitera-t-elle à l'Église? Cela dépendra de sa capacité à favoriser un processus "d'inculturation de la foi " qui prenne en compte les exigences rituelles des chrétiens. Le thème est à la mode. Il s'est trouvé au centre des débats des évêques africains lors de leur synode à Rome en septembre 1994. Saurons-nous donner une place aux essais timides des familles chrétiennes qui "inculturent " leur foi sans le savoir, rien qu'en la vivant, comme aux initiatives fulgurantes des prophètes populaires? Ou bien, "l'inculturation de la foi " devra-t-elle rester une façade, au même titre que «la démocratie » en politique? Pourtant l'enjeu vaut le risque d'une inculturation en profondeur. Et ce serait un bel exemple pour l'autre monde en mal de rites.

\section{Bibliographie}

LE GUÉRINEL, E.-N. 1987. «Psychanalyse en milieu africain? Le poids de la réalité culturelle ". Revue française de psychanalyse 1987/3 : p. 915 ss.

LEHMAN, Jean-Pierre. 1969. Le vécu corporel et ses interprétations en pathologie africaine. Abidjan, doc. polycopié. 